\title{
A circuit for simultaneous measurements of skin electrical conductance, susceptance, and potential
}

\author{
$\varnothing$ rjan G. Martinsen ${ }^{1,2,4}$, Håvard Kalvøy ${ }^{2}$, Dindar S. Bari ${ }^{3}$, and Christian Tronstad ${ }^{2}$ \\ 1. Department of Physics, University of Oslo, Oslo, Norway \\ 2. Department of Clinical and Biomedical Engineering, Oslo University Hospital, Norway \\ 3. Department of Physics, University of Zakho, Zakho, Kurdistan region, Iraq \\ 4. E-mail any correspondence to: ogm@fys.uio.no
}

\begin{abstract}
A circuit is presented that enables measurement of skin electrical conductance, susceptance, and potential simultaneously beneath the same monopolar electrode. Example measurements are shown to confirm the function of the circuit. The measurements are also in accordance with earlier findings that changes in skin conductance and potential do not always correspond and hence contain unique information.
\end{abstract}

Keywords: Electrodermal activity; bioimpedance; electrodes

\section{Introduction}

Electrodermal activity (EDA) has been studied for many decades and is typically measured as either skin conductance (at DC or low frequency $A C$ ) or skin potential [1,2]. Both parameters are sometimes measured and compared, but this has usually been done simultaneously contra-lateral (3) or in sequence (4).

In 2011, we described a set-up for simultaneous measurements of the two parameters with a constant current source and a two-electrode system where one of the electrodes were very large to enable quasi-monopolar measurements on the smaller electrode [5]. A few years later, our colleague, Prof. Sverre Grimnes, modified his three-electrode system from 1983 [6] to also enable simultaneous measurements of conductance, susceptance and potential, without the need for a large counterelectrode. This circuit was never published in detail, but has been used in a few publications [7-10]. Hence, in this article we will present the full circuit for the benefit of other research groups.

\section{Materials and methods}

The three-electrode circuit shown in figure 1 enables simultaneous measurement of skin potential, conductance, and susceptance. It is designed for connection to a data acquisition (DAQ) card that is interfaced to a computer. Hence, the interface connections to the DAQ card are for instance called AO_0 for analog output channel 0 or Al_0 for analog input channel 0.

Operational amplifier (opamp) U1 is connected as a Howland circuit, producing a current $i$ between the measuring electrode $\mathrm{M}$ and the current carrying electrode $\mathrm{C}$.

If all the resistors are equal (here $R=10 \mathrm{k} \Omega$ ), and the applied voltage is $200 \mathrm{mV}$ (amplitude) the current is given as:

$$
i=\frac{v}{R}=\frac{200 \mathrm{mV}}{10 \mathrm{k} \Omega}=20 \mu \mathrm{A}
$$

This current will flow through the M-electrode, which is placed on an electrodermally active skin site such as the hypothenar, through the tissue and to the C-electrode on the underarm. The current is measured by opamp U4 connected as a transresistance amplifier, so that the admittance values, conductance and susceptance, can be calculated by the computer software. This can be done by applying conventional lock-in technique, i.e. by multiplying the 
measured signal with the excitation signal (real part conductance) or by the excitation signal phase shifted 90 degrees (imaginary part - susceptance).

The voltage used when calculating the electrical admittance, is the potential difference between the $M$ electrode and a reference R-electrodes placed on a skin site with little or no electrodermal activity, such as the elbow.

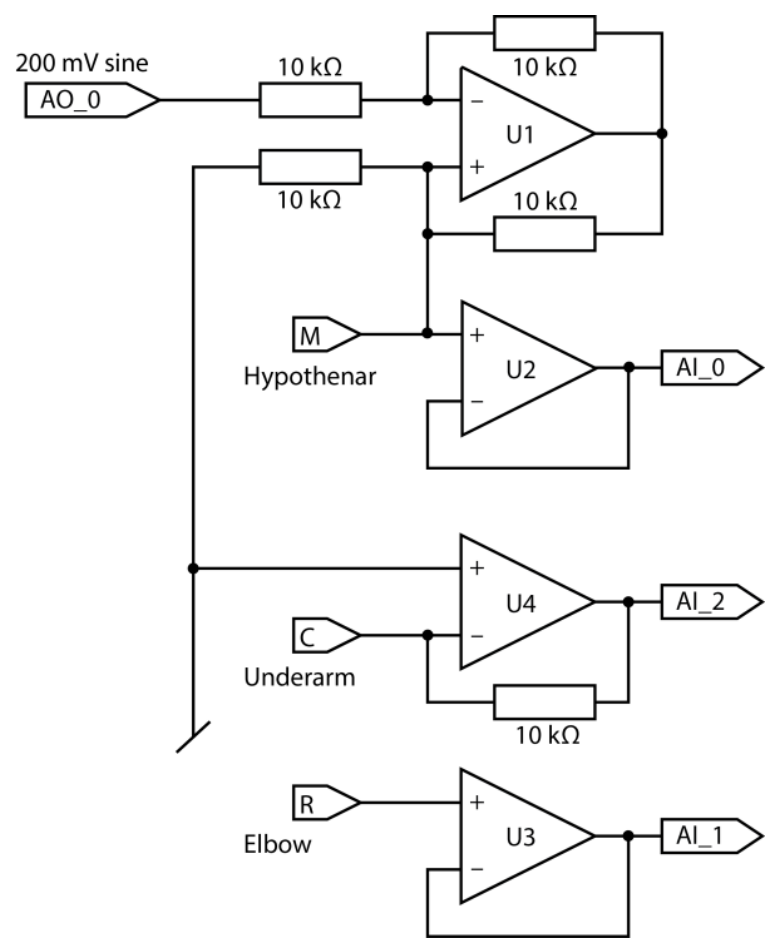

Figure 1: Circuit for simultaneous measurement of skin conductance, susceptance and potential.

The R-electrode is connected directly to the non-inverting input of opamp U3. Assuming no current flows to this input, Al_1 will then represent the potential of the deeper viable tissue. Consequently, the circuit will work as a threeelectrode system for quasi-monopolar measurements of the skin below the M-electrode (provided the frequency is low).

The skin potential is monitored by removing the measuring frequency in the $A / D$ converted voltage signal (AI_0 - Al_1) and looking at the fluctuations in the resulting DC offset.

As shown earlier, it is of utmost importance to use electrodes that do not fill the sweat ducts for this kind of measurements. This usually means that wet-gel electrodes cannot be used and that hydrogel or solid gel is preferred [11]. It is also important to keep the measurement frequency low to avoid influence from deeper, viable skin layers [12]. In this study, we have used a frequency of $20 \mathrm{~Hz}$.

\section{Measurements}

Measurements were done on two test subjects using KittyCat hydrogel electrodes (Kendall, KittyCat ${ }^{\circledR}, 1050$ NPSM). They were sitting isolated and comfortable in a temperature- controlled room $\left(22-23{ }^{\circ} \mathrm{C}\right)$ and were given three stimuli during the recording period: (1) they were asked to take a deep breath for 4 seconds; (2) they were given a mathematical task for 5 seconds (subtraction of 13 from $100)$; and (3) they watched a disturbing photo displayed for 3 seconds.

\section{Informed consent}

Informed consent has been obtained from all individuals included in this study.

\section{Ethical approval}

The research related to human use has been complied with all relevant national regulations, institutional policies and in accordance with the tenets of the Helsinki Declaration, and has been approved by the authors' institutional review board or equivalent committee.

\section{Results}

The results from measurements on the two test subjects are shown in figures 2 and 3 . The main peaks after approximately 50 seconds represent their reactions to the three stimuli. More details are given in the figure text.

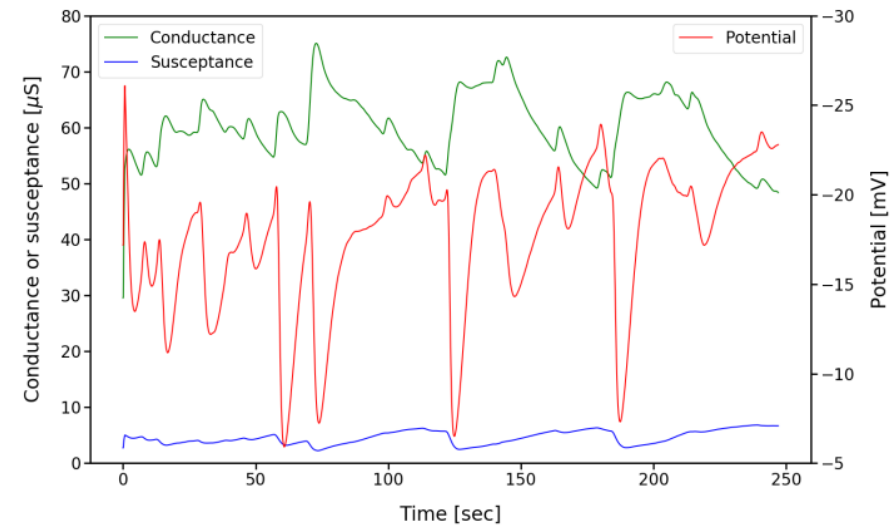

Fig.2: Measurements on a 20-year-old female healthy volunteer. Measured conductance in green, susceptance in blue and potential in red (right hand $\mathrm{Y}$-axis).

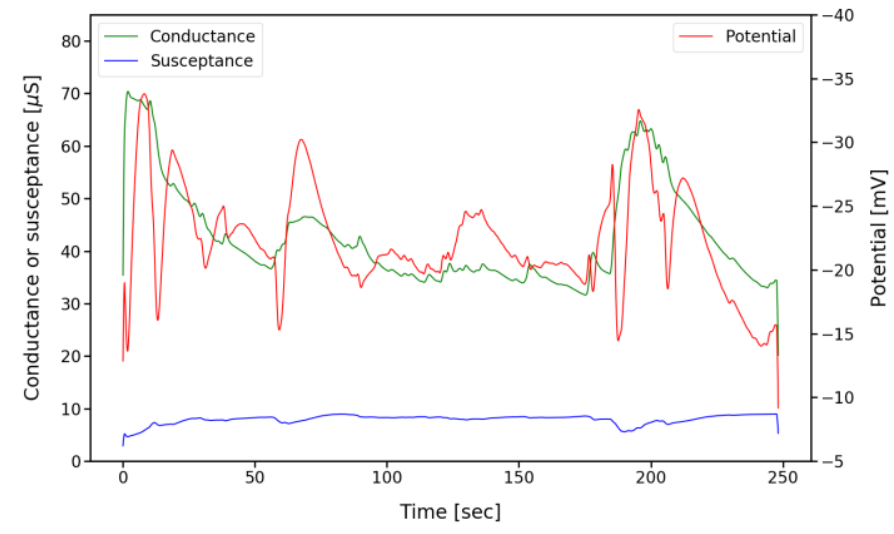

Fig.3: Measurements on a 19-year-old male healthy volunteer. Measured conductance in green, susceptance in blue and potential in red (right hand $\mathrm{Y}$-axis). 


\section{Discussion and conclusion}

We have presented a circuit that enables simultaneous measurements of electrical skin conductance, susceptance and potential with a three-electrode system. Results from test-measurements confirm that the circuit can produce measurements with good quality, and this has also been shown in earlier publications.

Changes in the low frequency skin susceptance has earlier been shown mainly to reflect the hydration level of the stratum corneum [13]. However, as can also be seen in figures 2 and 3, there are minor fluctuations also in the measured susceptance during the measurements period. Testing has shown that this is not due to poor channel separation between the real and imaginary part of the electrical admittance, and hence the underlying mechanism is not fully understood.

Skin potential often, but not always, follows the behavior of the skin conductance, thus confirming earlier findings that these two parameters represent different sources of information about skin physiology and electrodermal activity [14].

\section{Acknowledgements}

The authors acknowledge the seminal contribution from Prof. Sverre Grimnes in the development of instrumentation and methods for recording electrodermal activity in general and for the development of the circuit described in this article in particular.

\section{Conflict of interest}

Authors state no conflict of interest.

\section{References}

1. Gaviria B, Coyne L, Thetford PE. Correlation of skin potential and skin resistance measures. Psychophysiology. 1969;5:465477. https://doi.org/10.1111/j.1469-8986.1969.tb02850.x

2. Boucsein W. Electrodermal activity. Springer Nature AG, 2012.
3. Lykken DT, Miller RD, Strahan RF. Some properties of skin conductance and potential. Psychophysiology. 1968;5: 253268.4. https://doi.org/10.1111/j.1469-8986.1968.tb02821.x

4. Edelberg R, Burch NR. Skin resistance and galvanic skin response. Arch. Gen. Psychiatry. 1962;7:163-169. https://doi.org/10.1001/archpsyc.1962.01720030009002

5. Grimnes S., Jabbari A., Martinsen $\varnothing$.G., Tronstad C.: Electrodermal activity by DC potential and AC conductance measured simultaneously at the same skin site. Skin Res. Technol. 2011;17:26-34: https://doi.org/10.1111/j.1600-0846.2010.00459.x

6. Grimnes S. Impedance measurement of individual skin surface electrodes. Med. Biol. Eng. Comput. 1983;21:750755. https://doi.org/10.1007/bf02464038

7. Bari DS, Yacoob HY, Tronstad C, Kalvøy H, Martinsen $\varnothing \mathrm{G}$. Electrodermal responses to discrete stimuli measured by skin conductance, skin potential and skin susceptance. Skin Res. Technol. 2018;24:108-116. https://doi.org/10.1111/srt.12397

8. Bari DS, Yacoob HY, Tronstad C, Kalvøy H, Martinsen $\varnothing \mathrm{G}$. Influence of relative humidity on electrodermal levels and reponses. Skin Pharmacol. Physiol. 2018;31:298-307. https://doi.org/10.1159/000492275

9. Bari DS. Psychological correlates of nonspecific electrodermal responses. J. Electr. Bioimp. 2019;10:65-72. https://doi.org/10.2478/joeb-2019-0010

10. Tronstad C, Kalvøy H, Grimnes S, Martinsen $\varnothing \mathrm{G}$. Improved estimation of sweating based on electrical properties of skin. Ann. Biomed. Eng. 2013;41(5):1074-1083. https://doi.org/10.1007/s10439-013-0743-4

11. Tronstad C, Johnsen GK, Grimnes S, Martinsen $\varnothing$ G. A study on electrode gels for skin conductance measurements. Physiol. Meas. 2010;31:1395-1410. https://doi.org/10.1088/0967-3334/31/10/008

12. Martinsen $\varnothing \mathrm{G}$, Pabst $\mathrm{O}$, Tronstad C, Grimnes S. Sources of error in $\mathrm{AC}$ measurement of skin conductance. J. Electr. Bioimp. 2015;6:49-53. https://doi.org/10.5617/jeb.2640

13. Martinsen $\varnothing \mathrm{G}$, Grimnes $S$, Karlsen J. Electrical methods for skin moisture assessment. Skin Pharmacol. 1995;8(5):237245. https://doi.org/10.1159/000211353

14. Tronstad C, Kalvøy H, Grimnes S, Martinsen $\varnothing \mathrm{G}$. Waveform difference between skin conductance and skin potential responses in relation to electrical and evaporative properties of skin. Psychophysiology. 2013;50:1070-1078. https://doi.org/10.1111/psyp.12092 